\title{
A National Audit of Uveal Melanoma Referrals in Scotland
}

\author{
Niharika Nalagatla $\mathbb{C}^{1} \cdot$ Rhys $^{\text {Davies }^{2}} \cdot$ Julie Connolly $^{1} \cdot$ Paul Cauchi $^{1} \cdot$ Vikas Chadha $^{1}$
}

Received: 28 July 2020 / Revised: 9 January 2021 / Accepted: 14 January 2021 / Published online: 29 January 2021

(c) The Author(s), under exclusive licence to The Royal College of Ophthalmologists 2021

\section{To the Editor:}

The Royal College of Ophthalmologists (RCOphth) has published guidelines for referring patients with suspected adult ocular tumours in the UK [1]. In recent years, there has been a significant increase in referrals to all ocular oncology services (personal communication) but no evidence to indicate that the incidence of uveal melanoma is rising [2]. Data on the appropriateness of referrals is currently lacking. We carried out an audit of the referrals received by the Scottish Ocular Oncology Service (SOOS) from August 2017 to July 2018 to assess adherence to RCOphth guidelines.

Referrals for choroidal tumours, which included any group-A characteristic (thickness $>2.0 \mathrm{~mm}$, collar-stud configuration or documented growth) or two group-B characteristics (thickness $>1.5 \mathrm{~mm}$, presence of orange pigment, presence of serous retinal detachment or symptomatic patient), were regarded as satisfying the criteria [1].

Referrals for iris tumours had to contain at least one of the following to fulfil the criteria: tumour $>3.0 \mathrm{~mm}$ in diameter, markedly elevated tumour, presence of secondary glaucoma or cataract or tumour involving angle of the eye [1].

A total of 202 referrals from 26 hospitals were made over the 1-year period. Eighty-three per cent (168/202) were for choroidal lesions and 10\% (21/202) for iris lesions. Eleven referrals for choroidal lesions and 3 referrals for iris lesions were excluded from the study due to missing data.

Only 26\% (44/168) of referrals for choroidal lesions fulfilled the criteria. Forty-five per cent (20/44) of these patients were diagnosed with a malignant tumour and $18 \%$ (8/44) with an indeterminate lesion. Eighty-nine per cent

Niharika Nalagatla

niharika.nalagatla@nhs.net

1 Scottish Ocular Oncology Service, Tennent Institute of Ophthalmology, Glasgow, UK

2 Department of Ophthalmology, NHS Lothian, Edinburgh, UK
(39/44) needed treatment or monitoring at the SOOS and only $11 \%(5 / 44)$ were discharged after the first visit.

Sixty-seven per cent (113/168) of referrals for choroidal lesions had insufficient information; of these only 24\% (27/ 113) were diagnosed with a malignant lesion. Thirty-one per cent (35/113) were discharged from the service after the first visit.

Only $41 \%$ (69/168) had a B-scan and 15\% (26/168) of referrals mentioned the height of the lesion.

Thirty-eight per cent $(8 / 21)$ of patients referred with iris lesions met the criteria. Twenty-five per cent $(2 / 8)$ of these patients were found to have a melanoma and 50\% (4/8) had indeterminate lesions. Only $25 \%(2 / 8)$ were discharged after the first visit.

Forty-eight per cent (10/21) of referrals had insufficient information. None $(0 / 10)$ of these patients were found to have malignant tumours and 60\% (6/10) were discharged after the first visit.

The majority of referrals did not follow RCOphth guidelines. This audit demonstrates that adherence to guidelines can result in an increase in accuracy of referrals. Unnecessary referrals not only increase the burden on services but also potentially result in avoidable anxiety for patients. Many of these patients need to travel long distances to attend their appointment when a significant number could have been managed locally. All of this has become especially important in the current COVID-19 pandemic. A dedicated electronic referral form would prompt clinicians to consider whether sufficient information has been included and encourage utilisation of ultrasonography. Various versions of this form are now in use in a number of units and will be key in ensuring adherence to RCOphth guidelines.

Acknowledgements We would like to acknowledge the immense contribution of our ocular oncology coordinator, Ms Susan Ewan, in providing the administrative support for data collection.

Author contributions RD was responsible for the original study design, which was utilised locally, and data collection. VC was responsible for the study design for the national audit. NN was responsible for data collection and writing the article. JC, PC and VC provided guidance and feedback for the article. 


\section{Compliance with ethical standards}

Conflict of interest The authors declare that they have no conflict of interest.

Publisher's note Springer Nature remains neutral with regard to jurisdictional claims in published maps and institutional affiliations.

\section{References}

1. Rcophth.ac.uk. Referral pathways for adult ocular tumours 2019 [Internet]. 2020 [cited 29 May 2020]. Available from: https://www. rcophth.ac.uk/wp-content/uploads/2018/11/Referral-Pathways-forAdult-Ocular-Tumours-March-2019.pdf.

2. Kaliki S, Shields C. Uveal melanoma: relatively rare but deadly cancer. Eye. 2016;31:241-57. 